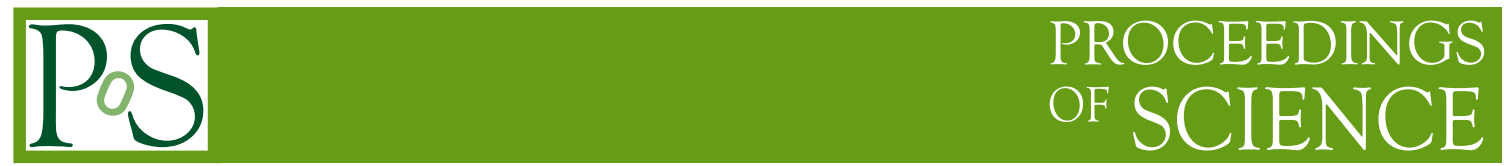

\title{
SU(2|1) chiral superfields and spinning models
}

\section{Stepan Sidorov ${ }^{a, *}$}

${ }^{a}$ Bogoliubov Laboratory of Theoretical Physics, Joint Institute for Nuclear Research, Joliot-Curie 6, 141980 Dubna, Moscow region, Russia

E-mail: sidorovstepan88@gmail.com

We consider the coupling of dynamical and semi-dynamical (spin) multiplets described by superfields living on the generalized $\mathrm{SU}(2 \mid 1), d=1$ chiral superspace. The interaction term of both multiplets is constructed as a superpotential term, where the dynamical multiplet is defined as a chiral multiplet $(\mathbf{2}, \mathbf{4}, \mathbf{2})$, while the semi-dynamical multiplet is associated with a mirror multiplet $(4,4,0)$.

RDP online workshop "Recent Advances in Mathematical Physics" - Regio2020,

5-6 December 2020

online

${ }^{*}$ Speaker 


\section{Introduction}

A new class of systems of $\mathcal{N}=4, d=1$ supersymmetric quantum mechanics called "Kähler oscillator" was introduced by S. Bellucci and A. Nersessian [1,2]. They studied supersymmetric oscillator models on Kähler manifolds with the term of the first-order in time derivatives responsible for the presence of a constant magnetic field. The bosonic Lagrangian of such system can be written as

$$
\mathcal{L}_{\text {bos. }}=g \dot{\bar{z}} \dot{\bar{z}}+\frac{i}{2} \mathbf{B}\left(\dot{z} \partial_{z} K-\dot{\bar{z}} \partial_{\bar{z}} K\right)-\omega^{2} g^{-1} \partial_{z} K \partial_{\bar{z}} K, \quad g=\partial_{z} \partial_{\bar{z}} K(z, \bar{z})
$$

It turned out, the presence of oscillator term and the interaction with a magnetic field deforms the standard $\mathcal{N}=4, d=1$ Poincaré supersymmetry to the so-called "Weak supersymmetry" [3]. We showed that the deformed superalgebra of the weak supersymmetry corresponds to the worldline supersymmetry $\mathrm{SU}(2 \mid 1)[4,5]$. We initiated a study of deformed supersymmetric quantum mechanics by employing superfield approach based on the worldline supersymmetry $\mathrm{SU}(2 \mid 1)$ with a mass dimension deformation parameter $m$.

Multiplets of $\mathcal{N}=4, d=1$ supersymmetry are denoted as $(\mathbf{k}, \mathbf{4}, \mathbf{4}-\mathbf{k})$ with $\mathbf{k}=\mathbf{0}, \mathbf{1}, \mathbf{2}, \mathbf{3}, \mathbf{4}$. These numbers correspond to the numbers of bosonic physical fields, fermionic physical fields and bosonic auxiliary fields. Wess-Zumino (WZ) type Lagrangians (sometimes referred as ChernSimons) for $(\mathbf{3}, \mathbf{4}, \mathbf{1})$ and $(\mathbf{4}, \mathbf{4}, \mathbf{0})$ were presented in the framework of the $\mathcal{N}=4, d=1$ harmonic superspace [6]. For example, the simplest WZ Lagrangian for $(\mathbf{4}, \mathbf{4 , 0})$ reads

$$
\mathcal{L}_{\mathrm{WZ}}=\frac{i}{2}\left(z^{i} \dot{\bar{z}}_{i}-\dot{z}^{i} \bar{z}_{i}\right)+\psi^{a} \bar{\psi}_{a}, \quad i=1,2, \quad a=1,2 .
$$

Without kinetic Lagrangian containing bosonic terms second-order in time derivatives, this Lagrangian describes a semi-dynamical multiplet. Fermionic fields become auxiliary, while bosonic fields satisfy the following Dirac brackets:

$$
\left\{z^{i}, \bar{z}_{j}\right\}=i \delta_{j}^{i}
$$

Such bosonic fields in supersymmetric quantum mechanics are usually called spin (isospin) variables.

Coupling of dynamical and semi-dynamical multiplets was proposed by [7]. This idea provided harmonic superfield construction of $\mathcal{N}=4$ extension of Calogero system with the additional spin degrees of freedom $z^{i}, z_{j}$. This work was followed by a further study of "spinning" models considering couplings of dynamical and semi-dynamical multiplets of various types (see, for example, [8] or [9]).

In this paper we briefly consider $\mathrm{SU}(2 \mid 1)$ superfield approach to spinning models ${ }^{1}$ based on the coupling of chiral superfields instead of harmonic ones [11].

\section{Superspace and superfields}

In this section we follow the notations introduced in [5].

\footnotetext{
${ }^{1}$ Undeformed version of these models was studied in [10]
} 
The superalgebra $\operatorname{su}(2 \mid 1)$ is defined as a deformation of the standard $\mathcal{N}=4, d=1$ Poincaré superalgebra:

$$
\begin{aligned}
& \left\{Q^{i}, \bar{Q}_{j}\right\}=2 \delta_{j}^{i} \mathcal{H}-2 m I_{j}^{i}, \\
& {\left[\mathcal{H}, Q^{k}\right]=-\frac{m}{2} Q^{k}, \quad\left[\mathcal{H}, \bar{Q}_{l}\right]=\frac{m}{2} \bar{Q}_{l},} \\
& {\left[I_{j}^{i}, \bar{Q}_{l}\right]=\frac{1}{2} \delta_{j}^{i} \bar{Q}_{l}-\delta_{l}^{i} \bar{Q}_{j}, \quad\left[I_{j}^{i}, Q^{k}\right]=\delta_{j}^{k} Q^{i}-\frac{1}{2} \delta_{j}^{i} Q^{k},} \\
& {\left[I_{j}^{i}, I_{l}^{k}\right]=\delta_{j}^{k} I_{l}^{i}-\delta_{l}^{i} I_{j}^{k} .}
\end{aligned}
$$

The indices $i, j(i=1,2)$ are $\mathrm{SU}(2)$ indices. The $\mathrm{U}(1)$ generator $\mathcal{H}$ is associated with the Hamiltonian. The generators $I_{j}^{i}\left(I_{k}^{k}=0\right)$ form $\mathrm{SU}(2)$ symmetry. In the limit $m=0$, models of the standard $\mathcal{N}=4$ supersymmetric mechanics are restored with $\mathcal{H}$ being a central charge generator.

\subsection{Generalized chiral superspace}

The $\mathrm{SU}(2 \mid 1)$ superspace is defined as a supercoset:

$$
\frac{\mathrm{SU}(2 \mid 1)}{\mathrm{SU}(2)} \sim \frac{\left\{\mathcal{H}, Q^{i}, \bar{Q}_{j}, I_{j}^{i}\right\}}{\left\{I_{j}^{i}\right\}}=\left\{t, \theta_{i}, \bar{\theta}^{j}\right\}
$$

It has a chiral subspace identified with the coset

$$
\frac{\left\{\mathcal{H}, Q^{i}, \bar{Q}_{j}, I_{j}^{i}\right\}}{\left\{\bar{Q}_{j}, I_{j}^{i}\right\}}=\left\{t_{\mathrm{L}}, \theta_{i}\right\} .
$$

The chiral condition reads

$$
\overline{\mathcal{D}}_{j} \Phi\left(t_{\mathrm{L}}, \theta_{i}\right)=0
$$

where $\overline{\mathcal{D}}_{j}$ and $\mathcal{D}^{i}$ are $\mathrm{SU}(2 \mid 1)$ covariant derivatives. A generalization of the chiral subspace is identified with the coset

$$
\frac{\left\{\mathcal{H}, \hat{Q}^{i}, \overline{\hat{Q}}_{j}, I_{j}^{i}\right\}}{\left\{\overline{\hat{Q}}_{j}, I_{j}^{i}\right\}}=\left\{\hat{t}_{\mathrm{L}}, \hat{\theta}_{i}\right\},
$$

where

$$
\hat{Q}^{i}=\cos \lambda Q^{i}-\sin \lambda \bar{Q}^{i}, \quad \overline{\hat{Q}}_{j}=\cos \lambda \bar{Q}_{j}+\sin \lambda Q_{j}
$$

The generalized chiral condition reads

$$
\left(\cos \lambda \overline{\mathcal{D}}_{j}-\sin \lambda \mathcal{D}_{j}\right) \Phi\left(\hat{t}_{\mathrm{L}}, \hat{\theta}_{i}\right)=0 .
$$




\subsection{Generalized chiral multiplet}

The generalized chiral condition describes a new type of the chiral multiplet $(\mathbf{2}, \mathbf{4}, \mathbf{2})$ defined on the generalized chiral superspace and depending on two deformation parameters: $m, \lambda$. Exactly this multiplet is a basis for the construction of supersymmetric Kähler oscillator models with the frequency of oscillator $\omega=m \sin 2 \lambda / 2$ and the strength of an external magnetic field $\mathbf{B}=m \cos 2 \lambda$. Both parameters disappear in the limit $m=0$ and the rotation parameter $\lambda$ becomes just an external automorphism parameter of the standard $\mathcal{N}=4$ Poincaré supersymmetry.

The chiral superfield is solved by

$$
\Phi\left(\hat{t}_{\mathrm{L}}, \hat{\theta}_{j}\right)=z+\sqrt{2} \hat{\theta}_{k} \xi^{k}+\hat{\theta}_{k} \hat{\theta}^{k} B
$$

where

$$
\hat{t}_{\mathrm{L}}=t+i \overline{\hat{\theta}}^{k} \hat{\theta}_{k}, \quad \hat{\theta}_{i}=\left(\cos \lambda \theta_{i} e^{\frac{i}{2} m t}+\sin \lambda \bar{\theta}_{i} e^{-\frac{i}{2} m t}\right)\left(1-\frac{m}{2} \bar{\theta}^{k} \theta_{k}\right) .
$$

Superfield invariant action for the chiral superfield $\Phi$ is given by

$$
S_{\text {kin. }}=\int d t \mathcal{L}_{\text {kin. }}=\frac{1}{4} \int d t d^{2} \theta d^{2} \bar{\theta}\left(1+2 m \bar{\theta}^{k} \theta_{k}\right) K(\Phi, \bar{\Phi})
$$

where $K(\Phi, \bar{\Phi})$ is a Kähler potential. Superpotential is given by the standard superfield action

$$
S_{\text {pot. }}=\int d \hat{t}_{\mathrm{L}} d^{2} \hat{\theta} f(\Phi)+\int d \hat{t}_{\mathrm{R}} d^{2} \overline{\hat{\theta}} \bar{f}(\bar{\Phi})
$$

\subsection{Mirror multiplet $(4,4,0)$}

The standard $\mathcal{N}=4$ multiplets have their mirror counterparts characterized by the interchange of two $\mathrm{SU}(2)$ groups which form $\mathrm{SU}(2) \times \mathrm{SU}^{\prime}(2) \rightarrow \mathrm{SO}(4)$ automorphism group of the standard $\mathcal{N}=4$ Poincaré supersymmetry [12]. Since this interchange $\left(i, j \longleftrightarrow i^{\prime}, j^{\prime}\right)$ has no essential impact on Poincaré supersymmetry, $\mathcal{N}=4$ multiplets and their mirror counterparts are mutually equivalent when dealing with only one multiplet from such a pair. Deformation to $S U(2 \mid 1)$ supersymmetry breaks the equivalence, because the first $\mathrm{SU}(2)$ group becomes subgroup of $\mathrm{SU}(2 \mid 1)$ and the second group $\mathrm{SU}^{\prime}(2)$ is broken. It means that $\mathrm{SU}(2 \mid 1)$ multiplets differ from their mirror counterparts.

The mirror $(\mathbf{4 , 4 , 0})$ satisfies the generalized constraints

$$
\overline{\tilde{D}}_{i} Y^{A}=0, \quad \tilde{\mathcal{D}}^{i} \bar{Y}^{A}=0, \quad \tilde{\mathcal{D}}_{i} Y^{A}=\overline{\tilde{D}}_{i} \bar{Y}^{A}, \quad \overline{\left(Y^{A}\right)}=\bar{Y}_{A},
$$

where

$$
\overline{\tilde{D}}_{i}=\cos \lambda \overline{\mathcal{D}}_{i}-\sin \lambda \mathcal{D}_{i}, \quad \tilde{\mathcal{D}}^{i}=\cos \lambda \mathcal{D}^{i}+\sin \lambda \overline{\mathcal{D}}^{i}
$$

The mirror multiplet is described by a pair of chiral superfields defined on (8) and satisfying the third condition. It gives the following simple solution:

$$
Y^{A}\left(\hat{t}_{\mathrm{L}}, \hat{\theta}_{i}\right)=y^{A}+\sqrt{2} \hat{\theta}_{i} \psi^{i A}+i \hat{\theta}_{k} \hat{\theta}^{k} \dot{\bar{y}}^{A}, \quad \overline{\left(y^{A}\right)}=\bar{y}_{A}, \quad \overline{\left(\psi^{i A}\right)}=\psi_{i A} .
$$


One can see that the last term is given by a time derivative of bosonic fields instead of auxiliary fields. So, when we write the superpotential action

$$
S_{\text {pot. }}=\frac{\mu}{2} \int d \hat{t}_{\mathrm{L}} d^{2} \hat{\theta} h\left(Y^{A}\right)+\frac{\mu}{2} \int d \hat{t}_{\mathrm{R}} d^{2} \overline{\hat{\theta}} \bar{h}\left(\bar{Y}_{A}\right),
$$

we obtain the WZ Lagrangian:

$$
S_{\text {pot. }}=\int d t \mathcal{L}_{\mathrm{WZ}}, \quad \mathcal{L}_{\mathrm{WZ}}=\mu\left[i \dot{\bar{y}}^{A} \partial_{A} h\left(y^{A}\right)+\frac{1}{2} \psi^{i A} \psi_{i}^{B} \partial_{A} \partial_{B} h\left(y^{A}\right)+\text { c.c. }\right] .
$$

\subsection{Gauged mirror multiplet $(4,4,0)$}

We can also assume that the chiral superfields $Y^{A}$ are subjected to the local $\mathrm{U}(1)$ transformations

$$
\left(Y^{1}\right)^{\prime}=e^{\frac{1}{2}(\Lambda-\bar{\Lambda})} Y^{1}, \quad\left(Y^{2}\right)^{\prime}=e^{-\frac{1}{2}(\Lambda-\bar{\Lambda})} Y^{2},
$$

where $\Lambda:=\Lambda\left(\hat{t}_{\mathrm{L}}, \hat{\theta}_{i}\right), \bar{\Lambda}:=\bar{\Lambda}\left(\hat{t}_{\mathrm{R}}, \overline{\hat{\theta}}^{j}\right)$. The superfields then satisfy the new gauge invariant constraints

$$
\begin{aligned}
& \left(\overline{\tilde{D}}^{i}+\frac{1}{2}\left[\overline{\tilde{D}}^{i}, X\right]\right) Y^{1}=0, \quad\left(\overline{\tilde{D}}^{i}-\frac{1}{2}\left[\overline{\tilde{D}}^{i}, X\right]\right) Y^{2}=0, \quad \text { c.c. } \\
& \left(\tilde{\mathcal{D}}^{i}-\frac{1}{2}\left[\tilde{\mathcal{D}}^{i}, X\right]\right) Y^{1}=\left(\overline{\tilde{D}}^{i}+\frac{1}{2}\left[\overline{\tilde{D}}^{i}, X\right]\right) \bar{Y}^{1} \\
& \left(\tilde{\mathcal{D}}^{i}+\frac{1}{2}\left[\tilde{\mathcal{D}}^{i}, X\right]\right) Y^{2}=\left(\tilde{\tilde{\mathcal{D}}}^{i}-\frac{1}{2}\left[\overline{\tilde{D}}^{i}, X\right]\right) \bar{Y}^{2}
\end{aligned}
$$

where the real superfield $X$ is a gauge superfield transforming as

$$
X^{\prime}=X+\Lambda+\bar{\Lambda} .
$$

According to the gauge invariant constraints it satisfies the additional constraint

$$
\tilde{\mathcal{D}}_{(i} \overline{\tilde{D}}_{j)} X=0 .
$$

It is solved by

$$
\begin{aligned}
X\left(t, \hat{\theta}_{i}, \overline{\hat{\theta}}^{j}\right)= & x+\sqrt{2}\left(\hat{\theta}_{k} \bar{\chi}^{k}+\overline{\hat{\theta}}^{k} \chi_{k}\right)+2 \overline{\hat{\theta}}_{k} \hat{\theta}^{k} \mathcal{A}+\hat{\theta}_{k} \hat{\theta}^{k} D+\overline{\hat{\theta}}^{k} \overline{\hat{\theta}}_{k} \bar{D} \\
& +\sqrt{2} i \overline{\hat{\theta}}^{k} \hat{\theta}_{k}\left(\hat{\theta}_{i} \dot{\bar{\chi}}^{i}-\overline{\hat{\theta}}^{i} \dot{\chi}_{i}\right)-\frac{1}{4} \hat{\theta}_{i} \hat{\theta}^{i} \overline{\hat{\theta}}^{j} \overline{\hat{\theta}}_{j} \ddot{x}, \\
& \overline{(x)}=x, \quad \overline{(\mathcal{A})}=\mathcal{A}, \quad \overline{(D)}=\bar{D}, \quad \overline{\left(\chi^{i}\right)}=\bar{\chi}_{i} .
\end{aligned}
$$

This superfield describes the mirror multiplet $(\mathbf{1}, \mathbf{4}, \mathbf{3})$ that differs from the ordinary one because of the deformation. Using the $\mathrm{U}(1)$ gauge freedom $X^{\prime}=X+\Lambda+\bar{\Lambda}$, we can choose the WZ gauge:

$$
X_{\mathrm{WZ}}=2 \overline{\hat{\theta}}^{k} \hat{\theta}_{k} \mathcal{A}, \quad \mathcal{A}^{\prime}(t)=\mathcal{A}(t)-\dot{\alpha}(t) .
$$

Thus, it can be interpreted as a mirror counterpart of the "topological" gauge multiplet described by the harmonic superfield $V^{++}$in the WZ gauge [13]. One can introduce accompanying chiral superfields

$$
\mathcal{V}_{\mathrm{WZ}}\left(\hat{t}_{\mathrm{L}}, \hat{\theta}_{i}\right)=\hat{\theta}_{k} \hat{\theta}^{k} \mathcal{A}, \quad \overline{\mathcal{V}}_{\mathrm{WZ}}\left(\hat{t}_{\mathrm{R}}, \overline{\hat{\theta}}^{j}\right)=\overline{\hat{\theta}}^{k} \overline{\hat{\theta}}_{k} \mathcal{A}
$$


satisfying

$$
\tilde{\mathcal{D}}_{i} X_{\mathrm{WZ}}=\overline{\tilde{\mathcal{D}}}_{i} \overline{\mathcal{V}}_{\mathrm{WZ}}, \quad \overline{\tilde{\mathfrak{D}}}_{i} X_{\mathrm{WZ}}=-\tilde{\mathcal{D}}_{i} \mathcal{V}_{\mathrm{WZ}}
$$

These superfields can be combined in the form of a triplet superfield $\mathcal{V}_{\mathrm{WZ}}^{\left(i^{\prime} j^{\prime}\right)}=\hat{\theta}^{k\left(i^{\prime}\right.} \hat{\theta}_{k}^{\left.j^{\prime}\right)} \mathcal{A}$ satisfying

$$
\tilde{\mathcal{D}}^{i\left(i^{\prime}\right.} \mathcal{V}_{\mathrm{WZ}}^{\left.j^{\prime} k^{\prime}\right)}=0
$$

Discarding the gauge fixing, this constraint describes the multiplet $(\mathbf{3}, \mathbf{4}, \mathbf{1})$.

According to the (anti)chiral conditions, the superfield solution is modified as

$$
\begin{aligned}
Y^{1}\left(t, \hat{\theta}_{i}, \overline{\hat{\theta}}^{j}\right) & =e^{-\frac{X}{2}} Y_{\mathrm{L}}^{1}\left(\hat{t}_{\mathrm{L}}, \hat{\theta}_{i}\right), & & \left(Y_{\mathrm{L}}^{1}\right)^{\prime}=e^{\Lambda} Y_{\mathrm{L}}^{1}, \\
Y^{2}\left(t, \hat{\theta}_{i}, \overline{\hat{\theta}}^{j}\right) & =e^{\frac{X}{2}} Y_{\mathrm{L}}^{2}\left(\hat{t}_{\mathrm{L}}, \hat{\theta}_{i}\right), & & \left(Y_{\mathrm{L}}^{2}\right)^{\prime}=e^{-\Lambda} Y_{\mathrm{L}}^{2} .
\end{aligned}
$$

Solving the additional constraint the left chiral superfield $Y_{\mathrm{L}}^{A}$ has the following $\theta$-expansion:

$$
Y_{\mathrm{L}}^{A}\left(\hat{t}_{\mathrm{L}}, \hat{\theta}_{i}\right)=y^{A}+\sqrt{2} \hat{\theta}_{i} \psi^{i A}+i \hat{\theta}_{k} \hat{\theta}^{k} \nabla_{t} \bar{y}^{A}, \quad \nabla_{t} \bar{y}^{1}=\left(\partial_{t}+i \mathcal{A}\right) \bar{y}^{1}, \quad \nabla_{t} \bar{y}^{2}=\left(\partial_{t}-i \mathcal{A}\right) \bar{y}^{2}
$$

Finally, the superpotential action must be written as a function of the only possible invariant $Y_{\mathrm{L}}^{1} Y_{\mathrm{L}}^{2}$, since it is the only gauge invariant object defined on the left chiral subspace.

\section{Coupling of dynamical and semi-dynamical multiplets}

We consider the model of the dynamical multiplet $(\mathbf{2}, \mathbf{4}, \mathbf{2})$ interacting with the semi-dynamical multiplet $(\mathbf{4 , 4 , 0})$. To couple these two multiplets we consider the simplest gauge invariant superpotential for them as

$$
S_{\text {int. }}=\frac{\mu}{2} \int d \hat{t}_{\mathrm{L}} d^{2} \hat{\theta} Y_{\mathrm{L}}^{1} Y_{\mathrm{L}}^{2} f(\Phi)-\frac{\mu}{2} \int d \hat{t}_{\mathrm{R}} d^{2} \hat{\hat{\theta}} \bar{Y}_{\mathrm{R}}^{1} \bar{Y}_{\mathrm{R}}^{2} \bar{f}(\bar{\Phi}),
$$

where $f$ is an arbitrary holomorphic function of $\Phi$. The component Lagrangian is then given by

$$
\begin{aligned}
\mathcal{L}_{\text {int. }}= & \mu\left[i\left(y^{1} \nabla_{t} \bar{y}_{1}-y^{2} \nabla_{t} \bar{y}_{2}\right) f+\psi^{i 1} \psi_{i}^{2} f+B y^{1} y^{2} \partial_{z} f\right. \\
& \left.+\xi^{i}\left(\psi_{i 1} y^{1}-\psi_{i 2} y^{2}\right) \partial_{z} f-\frac{\xi_{i} \xi^{i}}{2} y^{1} y^{2} \partial_{z} \partial_{z} f+\text { c.c. }\right] .
\end{aligned}
$$

We also construct the Fayet-Iliopoulos Lagrangian in chiral superspace

$$
S_{\mathrm{FI}}=-\frac{c}{4}\left[\int d \hat{t}_{\mathrm{L}} d^{2} \hat{\theta} \mathcal{V}_{\mathrm{WZ}}+\int d \hat{t}_{\mathrm{R}} d^{2} \overline{\hat{\theta}} \overline{\mathcal{V}}_{\mathrm{WZ}}\right] \Rightarrow \mathcal{L}_{\mathrm{FI}}=-c \mathcal{A}, \quad c=\text { const } .
$$

The total Lagrangian is a sum of these two terms and the kinetic Lagrangian (13) for the dynamical multiplet on a Kähler manifold.

The total Lagrangian is written as

$$
\mathcal{L}=\mathcal{L}_{\text {kin. }}+\mathcal{L}_{\text {int. }}+\mathcal{L}_{\text {FI }}
$$


Eliminating the auxiliary fields $B$ and $\psi^{i A}$ by their equations of motion and performing the following redefinition

$$
\begin{array}{lll}
y^{1}=v(f+\bar{f})^{-\frac{1}{2}}, & y^{2}=\bar{w}(f+\bar{f})^{-\frac{1}{2}}, & \xi^{i}=g^{-\frac{1}{2}} \eta^{i}, \\
\bar{y}_{1}=\bar{v}(f+\bar{f})^{-\frac{1}{2}}, & \bar{y}_{2}=w(f+\bar{f})^{-\frac{1}{2}}, & \bar{\xi}_{j}=g^{-\frac{1}{2}} \bar{\eta}_{j},
\end{array}
$$

we obtain the total on-shell Lagrangian (up to full time derivatives) as

$$
\begin{aligned}
\mathcal{L}= & g \dot{\bar{z} \dot{z}}+\frac{i}{2}\left(\eta^{i} \dot{\bar{\eta}}_{i}-\dot{\eta}^{i} \bar{\eta}_{i}\right)+\frac{i}{2} \mu(v \dot{\bar{v}}+w \dot{\bar{w}}-\dot{v} \bar{v}-\dot{w} \bar{w}) \\
& -\frac{i}{2} m \cos 2 \lambda\left(\dot{\bar{z}} \partial_{\bar{z}} K-\dot{z} \partial_{z} K\right)+\frac{i \mu\left(\dot{\bar{z}} \partial_{\bar{z}} \bar{f}-\dot{z} \partial_{z} f\right)}{2(f+\bar{f})}(v \bar{v}-w \bar{w}) \\
& +\frac{i}{2}\left(\dot{\bar{z}} \partial_{\bar{z}} g-\dot{z} \partial_{z} g\right) g^{-1} \eta^{k} \bar{\eta}_{k}-\frac{m \cos 2 \lambda}{2} \eta^{k} \bar{\eta}_{k}-\frac{\mu \partial_{z} f \partial_{\bar{z}} \bar{f}}{(f+\bar{f})^{2}}(v \bar{v}-w \bar{w}) g^{-1} \eta^{k} \bar{\eta}_{k} \\
& -\frac{\mu v \bar{w}}{(f+\bar{f})}\left[\frac{\partial_{z} \partial_{z} f}{2}-\frac{\partial_{z} f \partial_{z} f}{(f+\bar{f})}-\frac{\partial_{z} f}{2} g^{-1} \partial_{z} g\right] g^{-1} \eta_{i} \eta^{i} \\
& -\frac{\mu w \bar{v}}{(f+\bar{f})}\left[\frac{\partial_{\bar{z}} \partial_{\bar{z}} \bar{f}}{2}-\frac{\partial_{\bar{z}} \bar{f} \partial_{\bar{z}} \bar{f}}{(f+\bar{f})}-\frac{\partial_{\bar{z}} \bar{f}}{2} g^{-1} \partial_{\bar{z}} g\right] g^{-1} \bar{\eta}^{j} \bar{\eta}_{j} \\
& +\frac{m \sin 2 \lambda}{4}\left[\left(\partial_{z} \partial_{z} K-g^{-1} \partial_{z} K \partial_{z} g\right) g^{-1} \eta_{i} \eta^{i}+\left(\partial_{\bar{z}} \partial_{\bar{z}} K-g^{-1} \partial_{\bar{z}} K \partial_{\bar{z}} g\right) g^{-1} \bar{\eta}^{j} \bar{\eta}_{j}\right] \\
& -g^{-1}\left(\frac{\mu w \bar{v} \partial_{z} f}{(f+\bar{f})}-\frac{m \sin 2 \lambda}{2} \partial_{z} K\right)\left(\frac{\mu v \bar{w} \partial_{\bar{z}} \bar{f}}{(f+\bar{f})}-\frac{m \sin 2 \lambda}{2} \partial_{\bar{z}} K\right) \\
& +\frac{1}{4}\left(\partial_{z} \partial_{\bar{z}} g-g^{-1} \partial_{z} g \partial_{\bar{z}} g\right) g^{-2} \eta_{i} \eta^{i} \bar{\eta}^{j} \bar{\eta}_{j}+[\mu(v \bar{v}+w \bar{w})-c] \mathcal{A} .
\end{aligned}
$$

Looking at the last term, one concludes that the $\mathrm{U}(1)$ gauge field $\mathcal{A}$ plays the role of a Lagrange multiplier enforcing the constraint

$$
\mu(v \bar{v}+w \bar{w})-c=0 .
$$

Classical Hamiltonian reads

$$
\begin{aligned}
\mathcal{H}= & g^{-1} P_{z} P_{\bar{z}}-\frac{1}{4}\left(\partial_{z} \partial_{\bar{z}} g-g^{-1} \partial_{z} g \partial_{\bar{z}} g\right) g^{-2} \eta_{i} \eta^{i} \bar{\eta}^{j} \bar{\eta}_{j}+\frac{2 \partial_{z} f \partial_{\bar{z}} \bar{f} S_{3}}{g(f+\bar{f})^{2}} \eta^{k} \bar{\eta}_{k}+\frac{m \cos 2 \lambda}{2} \eta^{k} \bar{\eta}_{k} \\
& +\frac{S_{+}}{g(f+\bar{f})}\left[\frac{\partial_{z} \partial_{z} f}{2}-\frac{\partial_{z} f \partial_{z} f}{(f+\bar{f})}-\frac{\partial_{z} f}{2} g^{-1} \partial_{z} g\right] \eta_{k} \eta^{k} \\
& +\frac{S_{-}}{g(f+\bar{f})}\left[\frac{\partial_{\bar{z}} \partial_{\bar{z}} \bar{f}}{2}-\frac{\partial_{\bar{z}} \bar{f} \partial_{\bar{z}} \bar{f}}{(f+\bar{f})}-\frac{\partial_{\bar{z}} \bar{f}}{2} g^{-1} \partial_{\bar{z}} g\right] \bar{\eta}^{k} \bar{\eta}_{k} \\
& -\frac{m \sin 2 \lambda}{4}\left[\left(\partial_{z} \partial_{z} K-g^{-1} \partial_{z} K \partial_{z} g\right) g^{-1} \eta_{i} \eta^{i}+\left(\partial_{\bar{z}} \partial_{\bar{z}} K-g^{-1} \partial_{\bar{z}} K \partial_{\bar{z}} g\right) g^{-1} \bar{\eta}^{j} \bar{\eta}_{j}\right] \\
& +g^{-1}\left[\frac{\partial_{z} f S_{+}}{(f+\bar{f})}-\frac{m \sin 2 \lambda}{2} \partial_{z} K\right]\left[\frac{\partial_{\bar{z}} \bar{f} S_{-}}{(f+\bar{f})}-\frac{m \sin 2 \lambda}{2} \partial_{\bar{z}} K\right]
\end{aligned}
$$


where

$$
\begin{aligned}
& S_{3}=\frac{\mu}{2}(v \bar{v}-w \bar{w}), \quad S_{+}=\mu v \bar{w}, \quad S_{-}=\mu w \bar{v}, \\
& P_{z}=p_{z}-\frac{i}{2} m \cos 2 \lambda \partial_{z} K+\frac{i \partial_{z} f S_{3}}{(f+\bar{f})}+\frac{i}{2} g^{-1} \partial_{z} g \eta^{i} \bar{\eta}_{i}, \\
& P_{\bar{z}}=p_{\bar{z}}+\frac{i}{2} m \cos 2 \lambda \partial_{\bar{z}} K-\frac{i \partial_{\bar{z}} \bar{f} S_{3}}{(f+\bar{f})}-\frac{i}{2} g^{-1} \partial_{\bar{z}} g \eta^{j} \bar{\eta}_{j}
\end{aligned}
$$

Poisson (Dirac) brackets are imposed as

$$
\begin{array}{ll}
\left\{p_{z}, z\right\}=-1, & \left\{p_{\bar{z}}, \bar{z}\right\}=-1, \quad\left\{\eta^{i}, \bar{\eta}_{j}\right\}=-i \delta_{j}^{i}, \\
\{v, \bar{v}\}=i \mu^{-1}, & \{w, \bar{w}\}=i \mu^{-1} .
\end{array}
$$

\subsection{Spin variables}

The generators $S_{3}$ and $S_{ \pm}$, written trough spin variables, form the $s u(2)$ algebra:

$$
\left\{S_{3}, S_{ \pm}\right\}=\mp i S_{ \pm}, \quad\left\{S_{+}, S_{-}\right\}=-2 i S_{3}
$$

The Hamiltonian commutes with the Casimir operator

$$
C_{\mathrm{SU}(2)}=S_{+} S_{-}+\left(S_{3}\right)^{2}
$$

According to the constraint (37) the Casimir operator is determined by the constant

$$
C_{\mathrm{SU}(2)}=\frac{\mu^{2}(v \bar{v}+w \bar{w})^{2}}{4}=\frac{c^{2}}{4} .
$$

Its quantum counterpart (up to the ordering ambiguity) is given by ( $c \approx 2 s$ )

$$
C_{\mathrm{SU}(2)}=s(s+1) \text {, }
$$

where $s$ is a spin of the quantum system. Since the Hamiltonian commutes with the Casimir operator the spin of the system is preserved.

\section{Conclusions}

We proposed new models of SU(2|1) supersymmetric mechanics with the use of dynamical and gauged semi-dynamical multiplets. As an alternative of harmonic superspace, we exploited the generalized chiral superspace. We tried to obtain superconformal symmetry for considered models, but it is impossible at least for a single-particle model. Superconformal models can possibly be obtained for two or more chiral dynamical multiplets. It would be interesting to study mirror counterparts of the multiplets $(\mathbf{1}, \mathbf{4}, \mathbf{3})$ and $(\mathbf{3}, \mathbf{4}, \mathbf{1})$. The latter one is described by a triplet consisting of real and chiral superfields, i.e. $X, \mathcal{V}$ and $\overline{\mathcal{V}}$. For example we can couple the mirror multiplets $(\mathbf{3}, \mathbf{4}, \mathbf{1})$ and $(\mathbf{4 , 4 , 0})$ in $\mathrm{SU}(2 \mid 1)$ chiral superspace. 


\section{References}

[1] S. Bellucci, A. Nersessian, (Super)oscillator on $C P^{* * N}$ and constant magnetic field, Phys. Rev. D 67 (2003) 065013, [hep-th/0211070].

[2] S. Bellucci, A. Nersessian, Supersymmetric Kähler oscillator in a constant magnetic field, in proceedings of the workshop "Supersymmetries and Quantum Symmetries 2003”, SQS'03, 379-384 (2004).

[3] A.V. Smilga, Weak supersymmetry, Phys. Lett. B 585 (2004) 173, [hep-th/0311023].

[4] E. Ivanov, S. Sidorov, Deformed supersymmetric mechanics, Class. Quant. Grav. 31 (2014) 075013, [1307.7690 [hep-th]].

[5] E. Ivanov, S. Sidorov, Super Kähler oscillator from SU(2|1) superspace, J. Phys. A 47 (2014) 292002, [1312.6821 [hep-th]].

[6] E. Ivanov, O. Lechtenfeld, $N=4$ supersymmetric mechanics in harmonic superspace, JHEP 0309 (2003) 073, [hep-th/0307111].

[7] S. Fedoruk, E. Ivanov, O. Lechtenfeld, Supersymmetric Calogero models by gauging, Phys. Rev. D 79 (2009) 105015, [0812.4276 [hep-th]].

[8] S. Bellucci, S. Krivonos, A. Sutulin, Three dimensional N=4 supersymmetric mechanics with Wu-Yang monopole, Phys. Rev. D 81 (2010) 105026, [0911.3257 [hep-th]]

[9] E. Ivanov, M. Konyushikhin, A. Smilga, SQM with Non-Abelian Self-Dual Fields: Harmonic Superspace Description, JHEP 05 (2010) 033, [0912.3289 [hep-th]].

[10] S. Bellucci, N. Kozyrev, S. Krivonos, A. Sutulin, $N=4$ chiral supermultiplet interacting with a magnetic field, Phys. Rev. D 85 (2012) 065024, [1112.0763 [hep-th]].

[11] S. Sidorov, SU(2|1) supersymmetric spinning models of chiral superfields, J. Phys. A $\mathbf{5 4}$ (2021) 035205, [2003.01023 [hep-th]].

[12] E. Ivanov, J. Niederle, Bi-harmonic superspace for N=4 mechanics, Phys. Rev. D 80 (2009) 065027, [0905.3770 [hep-th]].

[13] F. Delduc, E. Ivanov, Gauging N=4 supersymmetric mechanics, Nucl. Phys. B 753 (2006) 211-241, [hep-th/0605211]. 